\title{
KONZEPT „LEHRER“ IN DEM PÄDAGOGISCHEN WELTBILD AUF DER GRUNDLAGE VON DEUTSCHEN PHRASEOLOGISMEN
}

\author{
Tetiana Kremenieva \\ $\mathrm{PhD}$, Assistent der Abteilung für Deutsche Philologie, \\ Oles Honchar Dnipro National University, Ukraine \\ e-mail: taniakremeneva@gmail.com,orcid.org/0000-0002-1933-900X
}

\section{Annotation}

Das Ziel dieser Forschung ist, phraseologische Einheiten in Form von Wortpaaren, Idiomen, Aphorismen und Sprichwörtern zu untersuchen und das sprachliche Weltbild des Lehrers als Fachsprachträgers zu veranschaulichen. Im Artikel wird die Stichprobe-Methode für Beschreibung der Lehrkompetenz, Arbeitsbedingungen und Beziehungen zwischen dem Lehrer und Lerner anhand der phraseologischen Einheiten sowie die quantitative Methode für Frequenzbestimmung von bei der Tätigkeit des Pädagogen verbalisierten Konzepten benutzt. Diese Forschung erzielt einen ausführlichen Überblick über die berufsbezogenen Kompetenzen des Lehrers und skizziert seine funktionale Bedeutung im Lernprozess. In den konzeptuellen Diskurs erlangen folgende Kompetenzen des Pädagogen wie eine fachliche, pädagogische, methodisch-didaktische, kommunikative, personale und Selbstkompetenz. Aufgrund der quantitativen Methode wurden die häufigsten Wörter in Wortpaaren, Idiomen, Aphorismen und Sprichwörtern festgestellt, was die nächsten konzeptuellen Modelle zu erstellen ermöglicht: „Lehrer ist Seele“ „Lehrer ist Wissen“ „Lehrer ist Schüler“, „Lehrer ist Augen“ „Lehrer ist Ohren“ „Lehrer ist Herz“ „Lehrer ist Gesicht“ „Lehrer ist Eltern“, „Lehrer ist Freude“ und „Lehrer ist Methode“. Mithilfe festgelegter Modelle kann die Funktionalität pädagogischer Tätigkeit im Bildungsprozess der Lerner anschaulich und aus kommunikativer Perspektive der Sprache erklärt werden.

Schlüsselwörter: Wortpaare, Idiome, Aphorismen, Sprichwörter, Lehrerkompetenz, Lehrerpersönlichkeit.

DOI https://doi.org/10.23856/3909

\section{Einführung}

In der heutigen anthropozentrischen Welt, wo der Mensch im Zentrum der wissenschaftlichen Forschung ist, wird eine besondere Aufmerksamkeit auf die Untersuchung der Korrelation zwischen menschlicher Sprache und Denken gerichtet. Bestimmte kognitive Mechanismen wecken das Interesse sowohl an den Werkzeugen für „Denkbildung und -ausdruck“ als auch der „Wissensordnung“ im menschlichen Gehirn (Boldyrev, 2004: 22). Im Kontext der Kognitionswissenschaft entsteht die Notwendigkeit, sich mit der Relevanz der fachbezogenen Denkstrukturen in einem bestimmten Tätigkeitsbereich auseinanderzusetzen. Im vorliegenden Artikel wird angeboten, sich durch die Sprache dem Lehrerberuf anzunähern. Dies scheint besonders wichtig angesichts dessen Aufgaben zu sein, die nicht nur Unterrichtsaktivitäten umfassen, sondern die Denkgestaltung der Lerner beinhalten. In Hinsicht auf wichtige mentale Prozesse, die mit erfolgreichem Lehr- und Lernprozess verbunden sind, ist Lehrerfachsprache von großer Bedeutung.

Das Ergebnis der kognitiven Tätigkeit vom Lehrer ist die Entstehung neuer „Berufseinheiten“für weitere,„Konzeptualisierung und Verbalisierung des Fachwissens“(Golovanova, 2013: 56). 
Dies führt dazu, dass die Wahrnehmung eines bestimmten Phänomens oder Objekts der Welt die Ursache für eine neue Informationsbeschaffung ist. Infolgedessen ermöglicht es, das Wissen auf der Grundlage eigener Kenntnisse und Erfahrungen zu bereichern.

Eine besondere Rolle bei der Fachwissensdarstellung der Fachleute spielen phraseologische Einheiten, die als eines der Kernprobleme in der kognitiven Phraseologie analysiert werden. Wie es bekannt ist, hat entstehendes phraseologisches Weltbild einen ,anthropozentrischen Charakter", mit dem die innere Welt des Lehrers, seine Persönlichkeit und Beziehung zur Außenwelt demonstriert werden können (Bagautdinova, 2006: 32). Heutzutage werden kognitive Linguistik, phraseologische Einheiten und kognitive Phraseologie von N. N. Boldyröw, V. V. Winogradow, Sch. M. Schtantschaew, G. A. Bagautdinowa, E. I. Golowanowa, O. A. Dmitriewa und anderen Forschern untersucht, es gibt aber keine Studie um das Konzept „Lehrer“ im Licht der phraseologischen Einheiten. Das Ziel der Arbeit besteht in der Analyse der phraseologischen Einheiten in der Fachsprache des Pädagogen, um das Abbild mit seinen Werten und seiner Weltanschauung zusammenzustellen. Die Stichprobe-Methode benutzend wurden Phraseologismen, Aphorismen und Sprichwörter aus den deutschen Fachtexten der Lehrer und anderen Quellen für diese Forschung ausgewählt. Die Relevanz dieser Untersuchung beruht auch auf der Tatsache, dass die Anzahl der Phraseologismen bezüglich des Begriffs „Pädagoge“ in den offiziellen Wörterbüchern minimal vorgestellt oder absolut abwesend ist.

\section{Klassifikationen der Phraseologismen}

Unter einem Phraseologismus versteht man ,stehende Wortverbindungen“, deren Bedeutung durch die einzelnen Komponenten ,nicht erfasst werden kann“(Vinogradov, 1986). Die Analyse der einzelnen Teile gibt uns keinen Einblick in ihre Bedeutung und außerdem kann diese beim Austausch der Komponenten verloren gehen. Darum ist der Phraseologismus die Zusammenbildung, die man nicht teilen oder austauschen kann.

Die wichtigsten phraseologischen Klassifikationen sind strukturell, semantisch, grammatisch, etymologisch, stilistisch und semantisch-strukturell. Die Klassifikation, die in der Publikation betrachtet wird, ist semantisch-strukturell, weil phraseologische Semantik eng mit der Studie der Persönlichkeit zu tun hat. Dementsprechend gibt es Wortpaare (Zwillingsformeln), Idiome, Aphorismen und Sprichwörter.

\section{Wortpaare (Zwillingsformeln)}

Wortpaare gelten als „,feste Wortverbindungen mit zwei Komponenten der gleichen Wortarten“ (Shtanchaev, 2009: 92). In den deutschsprachigen Fachtexten mit der pädagogischen Thematik sind häufige Wortpaare zu treffen, die verschiedene Kompetenzen präsentieren.

3.1. Fachkompetenz des Pädagogen in Form der Kenntnisse und Erfahrungen steckt in dem Wortpaar „Wissen und Können“: „Lehrer/innenexpertise zwischen Wissen und Können“ (Hascher, 2011). Der notwendige Teil des Lehrerberufs ist eine ständige Weiterentwicklung, die in dem Wortpaar „Fortbildung und Weiterbildung“6 angezeigt wird: „Lehrerinnen und Lehrer lernen: Fort- und Weiterbildung im Lehrerberuf“ (Richter, 2016).

3.2. Pädagogische Kompetenz bedeutet eine permanente Hingabe, Leidenschaft und Verantwortung in Wortpaaren „Mit Leib und Seele“: „Die Freude mit Kindern zu arbeiten, ihnen neben schulischen Dingen auch viel Praktisches fürs Leben mitgeben zu können, für sie auch bei Sorgen und Problemen da zu sein, hat mich nie an einen anderen Beruf denken lassen. Ich bin mit Leib und Seele Lehrerin“ (Bertelsmann, 2006). Das Wortpaar „Feuer 
und Flamme sein" ist auch voller Gefühle: "Am Anfang war ich mit Feuer und Flamme bei der Arbeit. Jetzt wächst mir alles über den Kopf“ (Zechmeister, 2010); „Chancen und Risiken“: „Chancen und Risiken, die sich aus Wahrnehmungen des Lehrer/ innen/handelns ergeben“ (Eichenberg, 2019); „Anspruch und Wirklichkeit": „Anspruch und Wirklichkeit des Wissensmanagement klaffen in der Praxis auseinander“ (Katenkamp, 2003); „Augen und Ohren“: „Mobbing der Grund sein, aber Eltern und Lehrer sollten in solch einer Situation Augen und Ohren offen halten und sich als Gesprächspartner anbieten “ (Christorf, 2017).

3.3. Selbstkompetenz des Pädagogen äußert sich in der angemessenen Verteilung der Berufs- und Freizeit im Wortpaar „Beruf und Freizeit": „Die bisherigen Seminare richteten sich an unterschiedliche Zielgruppen: an erfahrene Lehrkräfte, an Lehrkräfte 50plus, an Berufseinsteigerinnen und -einsteiger oder an Lehrkräfte in Ganztagsschulen. Allen gemeinsam war das Anliegen, die Teilnehmerinnen und Teilnehmer für das wichtige Gleichgewicht zwischen Beruf und Freizeit zu sensibilisieren " (Gigout, 2005).

\section{Idiome}

In der deutschen Sprache bilden Idiome die zahlreichste phraseologische Gruppe, die mit den Begriffen „Bildlichkeit“ und „Umdenken“ korrelieren (Shtanchaev, 2009). Die Übersetzung einzelner Teile und die wörtliche Übersetzung der Idiome ist sinnlos. Die Idiome in der Fachsprache des Lehrers präsentieren den Pädagogen als eine besondere Persönlichkeit.

4.1. Die positiven Charaktereigenschaften - Offenheit, Gutmütigkeit - drücken sich mit solchen Idiomen wie „Bock auf etwas haben“: „Lehrer sollen Bock auf Schule haben“ (Lehrer NRW, 5/2014), „ein Herz für etwas haben“: „Schülerinnen und Schüler müssen spüren, dass ihre Lehrerinnen und Lehrer ein Herz für sie haben “(Bremer Erklärung, 2000), „,mit Herzblut": „, Von der ersten Stunde an trete ich gern vor die Kinder und das mache ich heute noch mit genauso viel Herzblut wie vor 30 Jahren“ (Klasse, 2/2012), „warmherzig sein“: „Sämtliche Eigenschaften beziehen sich auf das Lernen der Schüler, also bedeutet warmherzig, dass den Lehrern das Lernen jedes Einzelnen persönlich am Herzen liegt“ (Bär, 2015), „ein offenes Ohr haben“: ,, Ich hoffe, dass ich für die Schüler ein Lehrer bin, der respektvoll und fair mit ihnen umgeht und auch bei Problemen ein offenes Ohr hat" (Klasse, 2/2012); Zurückhaltung in den Idiomen „Gesicht wahren“: , Wir haben weiter oben gesehen, dass die Lerner in der Regel bereit sind, den Lehrer aufzufangen und fachliche Defizite seinerseits diskret und taktvoll auszugleichen, so dass er sein Gesicht wahren kann“ (Buhlmann, 2000), „Gesicht sein“: , Lehrende sind letztlich das Gesicht von Weiterbildungsanbietern“ (Schratz, 2015) und Kontaktfreudigkeit in ,einen guten Draht haben“: „Ein guter Lehrer ist einer, der Kinder und Jugendliche mag und der einen guten Draht zu ihnen hat“ (Göppel, 2013), ,im Herzen jung bleiben“: „Ich versuche als älter werdender Lehrer, im Herzen jung zu bleiben und mir das Verständnis für die Jugend zu bewahren“ (Klasse, 2/2012) aus. Gleichzeitig können schwere Arbeitsbedingungen und Stress die Ursachen der negativen Emotion „Ärger“ sein: „Wir Lehrer sind sauer“ (Lehrer NRW, 2/2013).

4.2. Zu Lehrerkompetenzen zählt Fachkompetenz, die in zahlreichen den Idiomen vorgestellt wird. „Im Fach firm sein“": „Ein guter Lehrer ist einer, der in seinen Fächern firm ist“ (Buhlmann, 2000); „ein alter Hase sein“: „Lehrer in seinem Beruf ist erfahren und fühlt er sich jedoch alles andere als alter Hase" (Eichenberg, 2019); Erziehungskompetenz in „Augen öffnen“: „Lehrer, die die Leidenschaft haben, Kinder Augen zu öffnen“ (Klasse, 2/2012); Organisationskompetenz in ,im Griff haben“: „Lehrer haben ihre Klasse im Griff“ (Buhlmann, 2000); ,in der Hand behalten“, „im Blick behalten“: „, Der Lehrende muss bei 
Praxis das Zepter fest in der Hand und den Zweck klar im Blick behalten" (Lehrer NRW, 2/2013) und Unterstützungskompetenz in ,sich Zeit nehmen“: „Lehrer sollen mich unterstützen und sich Zeit nehmen " (Lehrer NRW, 2/2013).

4.3. Die Finanzlage wird oft zum Thema des Überlegens beim Lehrer, was in folgenden Idiomen widergespiegelt ist, wie ,über Wasser halten“: „Ihre schlecht bezahlten Lehrer, die sich mit Nebenjobs über Wasser hielten, hätten zudem kaum Zeit für die Schüler" (Lehrer NRW, 2/2013) und „arme Socken“: „Mit Fahnen, Plakaten und Flugblättern machten sie die Messebesucher auf das Problem aufmerksam. Das Motto der Aktion lautete: Angestellte Lehrkräfte-arme Socken “ (Lehrer NRW, 2/2013). Sie verweisen auf die schlechte Bezahlung und erbärmliche Situation des Pädagogen in der Gesellschaft mit der Notwendigkeit zu einem zweiten Standbein zu greifen. Das Idiom ,auf dem Schleudersitz sein“ im Satz „Unter dem Motto „Lehrer auf dem Schleudersitz“ lädt Lehrer am 14. März zum Vertrauenslehrertag ins Düsseldorfer Hotel Nikko ein “ (Lehrer NRW, 1/2014) definiert den Lehrerberuf als Beruf mit vielen Nachteilen und Problemen. Das ist eine Enttäuschung und Frustration für Lehrer und kann in diesem Fall als Kündigung enden. Die schweren, mit Stress verbundenen Arbeitsbedingungen werden in den Idiomen , unter Druck sein“ dargestellt: „immer öfter werden Lehrer von Eltern unter Druck gesetzt“ (Lehrer NRW, 1/2014); „an ihre Grenzen geraten“: „Insbesondere junge Lehrkräfte geraten im System Schule schnell an ihre Grenzen“ (Lehrer NRW, 1/2020).

\section{Aphorismen}

Das Bild des Pädagogen kann durch Aphorismen in komprimierter Form verfolgt werden, weil sie „Ideen“ und „Stimmungen“ der bestimmten Nation vertreten (Dmitrieva, 1997: 16). Sie kennzeichnen sich durch „Originalität des Denkens“ und „semantische Vollständigkeit“, mittels welcher man die Individualität des Lehrers erforschen kann (Dmitrieva, 1997: 18). Im Gegensatz zu Sprichwörtern streben sich Aphorismen ,nach Wahrheit" und darum ermöglichen die Entdeckung sowohl der positiven als auch negativen Aspekte des Lehrerberufs. Die Persönlichkeit des Pädagogen wird in folgenden Aphorismen realisiert wie:

5.1. Fachkompetenz. Einer der wichtigsten Werte des Pädagogen ist Lehrfähigkeit, die durch die Leidenschaft und Motivierung erreicht werden kann. Die Bereitschaft zur Kommunikation und Interaktion mit Lernern wird auch oft in den Aphorismen betont. Ein Beispiel ist „Ein Lehrer ohne Freudigkeit ist eine Uhr ohne Feder“, wo der Lehrer ohne Leidenschaft mit einer Uhr ohne Feder verglichen wird. Der Lehrer wird zum Kompass, der den Lernenden im Leben die Richtung weist. Die Verfügbarkeit der Erfahrungen und Fachwissen ist im Aphorismus „Lehrer sind Fixer: sie denken immer nur an den Stoff“, solange der kompetente Lehrer Lernmaterialien wählen, ändern, bearbeiten und unterrichten müssen. Andere Lehrerspflichte sind Wissensvermittlung im Aphorismus „, Gute Lehrer sind Fleißverkäufer“ mit der Neuinterpretation vom Lehrer als Vermittler guter Lehrstoffe in der Form von Wissen und Denkbildung der Lerner in „Die schwierigste Aufgabe eines Lehrers ist es nicht, Wissen zu vermitteln, sondern zu eigenständigem Denken zu befähigen “.

5.2. Methodisch-didaktische Kompetenz. Das Aphorismus „Jeder Lehrer muss seine eigene Methode haben, er muss sie sich mit Verstande erschaffen haben, sonst fromme er nicht; ein blinder Führer der Blinden " weist auf die Wichtigkeit eigener Methoden für den Unterricht und die Erziehung der Schüler hin. Wenn es nicht so passiert, verwandelt sich der Lehrer in einen blinden Menschen, der ,keinen Weg“ sieht. Die Lerner sind auch „,blind“ ohne Vorstellung über eigene Lerntechniken und Lernziele. In einem anderen Beispiel „Ein Lehrer ohne Methode ist ein Komponist ohne Generalbass, ein Virtus ohne Takt“ sind Methodenkenntnisse 
einer großen Bedeutung für Lehrerkompetenz wie Generalbass und Takt für Musiker. Der Lerner wird ein „Meisterwerk“ vom Lehrer, wenn der letzte über methodische Materialien verfügt. Dafür braucht man viel Kreativität ohne Langeweile während des Unterrichts: «Ein guter Ausbilder muss keine Schleifmaschine sein“.

5.3. Kommunikative Kompetenz. Der Interaktionserfolg zwischen dem Lehrer und den Lernern hängt von der Fähigkeit zur Empathie ab. Der Pädagoge muss in das Leben seiner Studenten eintauchen und „Freund“ und „Klassenkamerad“ für seine Lerner werden: „Pädagogen sind Beziehungskatalysatoren“, „Kein Lehrer, der nicht auch Schüler sein kann “, „Lehrer sind Mitschüler“. Der Aphorismus „Das Verhältnis des wahren Lehrers zum Schüler ist ein innerliches, ein geheimnisvolles, ein unmittelbares von Seele zu Seele. Sie tauschen die Seelen gegeneinander aus " betont einen nicht sichtlichen, aber sehr empfindlichen Faden im Lehrer-Student-Verhältnis beim Lernprozess. Solche Interaktion tut gut nicht nur für Wissen, Persönlichkeitsentwicklung und Weltanschauung des Lerners, sondern auch des Lehrers. Die Schüler selbst können „Lehrer“" werden und etwas Neues lernen: „Die besten Lehrer sind Vorlerner“, „Erfolgreiche Lehrer sind Schüler ihrer Schüler“, „Den guten Lehrer machen nicht selten die guten Schüler“, „Schulstunden sind Nachhilfestunden für Lehrer“.

5.4. Pädagogische Kompetenz gliedert sich in psychologische, erzieherische und organisatorische Fähigkeiten bei der Arbeit mit Schülern. Es gibt Pflichten, die von Pädagogen erfüllt werden müssen: „Lehrer haben vormittags Recht und nachmittags frei“. Die Lehreraufgabe hier ist Lernziele anzusprechen: „,Wer unterrichten will, muss das Ziel, zu dem er die Schüler führen will, kennen und Weg ganz überschauen, um dahin zu gelangen“; eine Person mit Moral für künftige Weltveränderungen zu erziehen: „Ein Lehrer arbeitet für die Ewigkeit. Niemand kann sagen, wo sein Einfluss endet"; Weltanschauungs- und Persönlichkeitsbildung mit seinem eigenen Vorbild: „Nur schlechte Lehrer haben schlechte Schüler“, „Wie der Lehrer, so die Schüler", „Einen schlechten Lehrer imitieren oder vergessen seine Schüler, einen guten Lehrer übertreffen sie “. Der Lernprozess erfordert ständige Kontrolle und Organisation, die im Aphorismus „Das lebendige Auge des Lehrers ist der beste Regulator der ganzen Klasse“ausgedrückt werden und es kostet die Lehrer viel Arbeit und Mühe. In den Aphorismen wie „Der Lehrer ist nie fertig“ und ,Erziehung ist eine Wanderung, keine Flugreise” wird gemeint, dass Lehrerleben ziemlich stressig und problematisch ist.

5.5. Persönliche Kompetenz. Der Lehrerberuf erfordert bestimmte Charaktereigenschaften für erfolgreiche Interaktion mit Lernern. Der Aphorismus „Auf die Persönlichkeit des Lehrers kommt alles an. Der Wert aller Methoden und Verordnungen ist zweifelhaft “ zeigt, dass die Bedeutung der Lehrerpersönlichkeit für Lehren und Lernen nicht zu unterschätzen ist. Außerdem bilden sich Weltanschauung und Denken der Lerner wie die nötigen Aspekte für weitere Persönlichkeitsbildung: „Des Lehrers schönstes Vorrecht ist, dass er nicht nur durch sein Wissen, auch durch seine Persönlichkeit die Seelen der nächsten Generation adelt“. Freude und Humor sind zwei wichtige Charaktereigenschaften der Lehrer für Kommunikation mit seinen Lernern ohne Barrieren und, also, eine erfolgreiche Wissensvermittlung: „Unmotivierte Schüler brauchen keine Pädagogen, die ihnen immer mehr Wissen vermitteln, sondern mehr Freude am Lernen“; „Es ist schön, wenn ein Lehrer Humor hat. Humor ist Feuchtigkeit und nichts braucht die Schulluft nötiger".

\section{Sprichwörter}

Während der Recherchen wurde sich herausgestellt, dass es seltene Sprichwörter in der deutschen Sprache in Bezug auf lehrerbezogene Tätigkeit gibt. Sie berühren aber die sakrale 
Tiefe im Lehrer-Student-Verhältnis, stellend die Person des Lehrers den Eltern und sogar dem Gott gleich: „, Wer dich einen Tag unterrichtete, ist das ganze Leben lang dein Vater“; „, Verehre deine Lehrer wie deine Eltern“; „Gott, Eltern und Lehrer kann man nie vergelten“. Gleichzeitig das Sprichwort „Wen Gott im Zorn schuf, macht er zum Lehrer“ enttarnt die Pflicht des Pädagogen als eine große Belastung.

\section{Resultate}

Während der Untersuchung wurden die phraseologischen Einheiten der Verbalisierung von wichtigsten Äußerungen der Lehrertätigkeit wie Lehrerkompetenz, Beziehungen und Arbeitsbedingungen festgestellt.

Bereich 1 - Lehrerkompetenz beinhaltet Subbereiche wie Fachkompetenz mit Konzepten „Wissen“, „Können“, „Lehrfähigkeit", „Wissensvermittlung“" und „Denkbildung“; didaktisch-methodische Kompetenz mit Lehrmethodik“ und „Kreativität"; pädagogische Kompetenz teilt sich in Konzepte „Pflicht", „Zielsetzung“6, „Weltanschauungsbildung“, „Vorbild“", „Verantwortung“", „Kontrolle“6 und „Beobachtung“; persönliche Kompetenz besteht aus solchen Konzepten wie „Liebe“, „Freude“6, „Kontaktfreudigkeit“, „Humor", „Gutmütigkeit", ,Selbstkontrolle“", aber auch „Ärger“6; Selbstkompetenz mit Konzepten „Weiterentwicklung“", „Lernkompetenz", „Fortbildung“ und „,Work-Life Balance“.

Bereich 2 - Beziehungen äußert sich in Konzepten um die Beziehungen zwischen dem Lehrer und Lerner. Die Beziehung zum Pädagogen realisiert sich in Konzepten „Vater“6, „Eltern“ und „Gott“6. Die Konzepte „Schüler“6 und „Mitschüler“6 betrachten eine gerechte Behandlung des Lerners ohne Amtsmissbrauch von der Seite der Pädagogen.

Bereich 3 - Arbeitsbedingungen. Die Konzepte „Armut", „Druck ${ }^{66}$, ,Stress „Zwecklosigkeit"6 und „Gottesgericht" in Idiomen, Aphorismen und Sprichwörtern stellen ein klares Bild über eine schwere Arbeit der Lehrer mit unzureichenden Arbeitsbedingungen und einem geringen Gehalt.

Nach dem Häufigkeitsgebrauch von phraseologischen Einheiten kann man folgende Konzepte im Zusammenhang mit der Fachkommunikation der Lehrer feststellen:

Tabelle 1. Konzeptsphäre der Lehrerpersönlichkeit

\begin{tabular}{|c|c|c|c|}
\hline \multirow{2}{*}{ № } & GEBIET & KONZEPT & ANZAHL \\
\hline \multirow{3}{*}{1.} & \multirow{3}{*}{ KÖRPERTEIL } & AUGEN & 4 \\
\cline { 3 - 4 } & & HERZ & 3 \\
\cline { 3 - 4 } & & OHREN & 2 \\
\cline { 3 - 4 } & \multirow{2}{*}{2.} & GESICHT & 4 \\
\hline \multirow{2}{*}{3.} & \multirow{2}{*}{ GEISTLICHES GEBIET } & SCHÜLER & 2 \\
\hline 4. & ZUSTAND & ELTERN & 5 \\
\hline 5. & \multirow{2}{*}{ ANDERS } & SEELE & 2 \\
\cline { 3 - 4 } & & FISUDEN & 4 \\
\hline
\end{tabular}

Die häufigsten Konzepte könnten folgendermaßen interpretiert werden: „Lehrer ist Seele“ zeigt den Lehrer als Hauptkraft des Lernerlebens und Haupthebel deren Denkens-, 
Gefühls- und Wissensbildung; „Lehrer ist Wissen“ als eine Hauptquelle der Wissenserwerbung von Lernern; „Lehrer ist Schüler“, der immer strebt, neue Kenntnisse zu sammeln und freundliche Beziehungen zu seinen Lernern zu unterstützen; „Lehrer ist Augen“ und „Lehrer ist Ohren“, die ständig Situation während des Lernprozesses kontrollieren; „Lehrer ist Herz“ wie ein Symbol für Empathie, Mitgefühl und Gutmütigkeit; „Lehrer ist Gesicht“ wie ein Vorbild für seine Lerner; „Lehrer ist Eltern“, der sich um Kinder kümmert, wie ein Vater oder eine Mutter; „Lehrer ist Freude“ und „Lehrer ist Methode“ als Begeisterungsmittel und Motivation zum Lernen.

Das Erlernen des sprachlichen Weltbilds des Pädagogen mit Hilfe von Phraseologismen, Aphorismen und Sprichwörtern ermöglicht zwar nicht vollständig, aber ziemlich grundsätzlich die beim Lehrprozess entstandenen und durch Lehrertätigkeit eingeprägten Denkmodelle zu erklären. Die nächsten Untersuchungen sollten einen festen Kernbestandteil sowie mit der Zeit variierende sprachliche Einheiten in der Lehrerfachsprache entdecken und erklären.

\section{Referenz}

Bagautdinova G. A. (2006). Frazeologismy antropocentricheskoy napravlennosti i yazykovaya kartina mira [Idioms of anthropocentric direction and linguistic worldview]. Kazan: Centr innovacionnyh technologij. [In Russian]

Boldyrev N. N. (2004). Konzeptualnoe prostranstvo kognitivnoj lingvistiki [The conceptual space of cognitive linguistics]. Voprosy kognitivnoj lingvistiki. [In Russian]

Dmitrieva O. A. (1997). Kulturno-yazykovye harakteristiki poslovic i aforismov [Cultural and linguistic peculiarities of proverbs and aphorisms]. Volgograd: 10.02.20. [In Russian]

Golovanova E. I. (2013). Frazeologism kak osobyj tip terminologicheskoj nominacii [Idioms as a special type of nomination]. Kyiv: Terminologichnyi visnyk. [In Russian]

Vinogradov V. V. (1986). Ob osnovnyh tipah frazeologicheskih edinic v russkom yazyke [About main types of phraseological units in the Russian language]. Moskva: Nauka. [In Russian]

Shtanchaev Sh. M. (2009). Strukturno-semanticheskaya klassifikaciya frazeologizmov nemeckogo yazyka [Structural-semantic classification of idioms in German language]. Obshhecnvennye gumanitarnye nauki. [In Russian]

Bär M. (2015). Die Lehrerrolle - ein Überblick über eine unübersichtliche Landschaft. Bayern: RPZ-Impulse.

Bertelsmann-Stiftung \& Gewerkschaft Erziehung und Wissenschaft (Hrsg.) (2006). Lust auf Schule. Modul Lehrkräftegesundheit. Gütersloh: Bertelsmann.

Bremer Erklärung (2000). Aufgaben von Lehrerinnen und Lehrern heute - Fachleute für das Lernen. Gemeinsame Erklärung des Präsidenten der Kultusministerkonferenz und der Vorsitzenden der Bildungs- und Lehrergewerkschaften sowie ihrer Spitzenorganisationen Deutscher Gewerkschaftsbund DGB und DBB - Beamtenbund und Tarifunion. Zugleich Beschluss der Kultusministerkonferenz vom 5.10.2000. http://www.kmk.org/ doc/publ/erklaerung.pdf.

Buhlmann R., Fearns A. A. (2000). Handbuch des Fachsprachenunterrichts. Tübingen: Narr. (Neuauflage 2017: Berlin: Frank und Timme).

Christorf E., Gerhartz-Reiter S. (2017). Lehrer/innenhandeln wirkt - zu den Chancen und Risiken schulischer Interaktion. Innsbruck: Studien Verlag.

Eichenberg C. Auersperg F. (2019). Chancen und Risiken digitaler Medien für Kinder und Jugendliche. Göttingen: Hogrefe Verlag GmbH \& Co.KG.

Gigout F. (2005). Work-Life-Balance Im Lehrerberuf Schule, Privates und Soziales unter einen Hut bringen. Heidesheim: ADIS. 
Göppel R. (2013). Was macht eine gute Lehrerin, einen guten Lehrer aus? - Was kennzeichnet gute LehrerInnenbildung? Und was sollen Lehramtsstudierende speziell im erziehungswissenschaftlichen Studium lernen? .

Hascher T., Gruber H. (2011). Lehrer/innenexpertise zwischen Wissen und Können. Weinheim: Beltz Juventa.

Katenkamp O. (2003). „Die Praxis des Wissensmanagements: aktuelle Konzepte und Befunde in Wirtschaft und Wissenschaft. Münster: LID.

Klasse. Das Magazin für Schule in Sachsen (2/2012). Sachsen: Vetters GmbH \& Co. KG.

Lehrer NRW. Verband für den Sekundarbereich. (2/2013). Düsseldorf: Pädagogik \& HOCHSCHUL VERLAG.

Lehrer NRW. Verband für den Sekundarbereich. (1/2014). Düsseldorf: Pädagogik \& HOCHSCHUL VERLAG.

Lehrer NRW. Verband für den Sekundarbereich. (5/2014). Düsseldorf: Pädagogik \& HOCHSCHUL VERLAG.

Lehrer NRW. Verband für den Sekundarbereich. (1/2020). Düsseldorf: Pädagogik \& HOCHSCHUL VERLAG.

News4Teachers. Das Bildungsmagazin (4/2016).

Richter D. (2016). Lehrerinnen und Lehrer lernen: Fort- und Weiterbildung im Lehrerberuf. Münster; New York: Waxmann.

Schratz M., Schrittesser I. (2015). Was müssen Lehrerinnen und Lehrer in Zukunft wissen und können? Baltmannsweiler: Schneider Hohengehren

Zechmeister K. (2010). Burnout bei Lehrern. Möglichkeiten der Hilfestellung. Wien: Stand.

\section{Referenzen von Aphorismen und Sprichwörtern}

Aphorismen, Zitate, Sprüche und Gedichte: https://www.aphorismen.de/ suche?f_thema=Lehrer. Lehrer - Zitate: https://www.quotez.net/german/lehrer.htm. 